International Journal of Distributed and Parallel Systems (IJDPS) Vol.4, No.1, January 2013

\title{
A Review on Data Collection Method With SINK NODE IN WIRELESS SENSOR NETWORK
}

\author{
Suchita R.Wankhade ${ }^{1}$ and Nekita A.Chavhan ${ }^{2}$ \\ ${ }^{1}$ Department of Computer Science and Engineering, G. H. Raisoni College of \\ Engineering, Nagpur, India \\ suchita2007@gmail.com \\ ${ }^{2}$ Department of Computer Science and Engineering, M.E (Wireless Communication and \\ Computing), G. H. Raisoni college of Engineering, Nagpur, India \\ nikichavan@gmail.com
}

\begin{abstract}
Wireless sensor networks are highly distributed of small, lightweight wireless nodes. In wireless sensor network, the sensor nodes are distributed in various geographical dispersed areas for sensing the data and the sink node is used to collect data from different sensor nodes, therefore data collection is important issue in wireless sensor network. Every node in sensor network consist of three subsystem, first sensor subsystem which sense environment, second processing subsystem which perform local computation on sensed data and third communication subsystem which is responsible for message exchange. Various Studies in this field exploit that mobile sink node is used to collect environmental observations such as weather forecasting data from sensor nodes. This review paper first shows the WSNs with sink node and their architecture then shows the comparative study of different sensor data collection method and sink node data collection method it then decides which technique is efficient for the data collection from the sensor nodes.
\end{abstract}

\section{KEYWORDS}

Wireless sensor networks (WSNs), Sink node, Mobile sink node, Data base, Data collection.

\section{INTRODUCTION}

Wireless sensor networks have been applied to many applications and sensor nodes are used in variety of application such as military application of sensor node battlefield surveillance and area monitoring, commercial application at home and industries, event detection (intrusion/flood emergencies) etc. Among them, one of the most important applications is sensor data collection, where sensed data is continuously collected at all or some of the sensor nodes and forwarded through wireless communications to a central base station for further processing.

Recently, there is many increasing interest on data collection in wireless sensor network. Sink node is used to collect data in wireless sensor network; data collection may one hop, multi- hop, all sensor collect data is send to the base station called sink node.

Large or thousand numbers of Sensor nodes deployed in collaborate to form a network capable of reporting to data collection and deployment can be random deployment or self organization. In some cases mobile sink node move or travel in sensing area and directly collects data from the sensors and send to the base station for further processing. Taxi, Airplane, bus is used for the data collection. This may be reducing communication traffic by using mobile sink node data collection.

DOI : $10.5121 / \mathrm{ijdps} .2013 .4106$ 
International Journal of Distributed and Parallel Systems (IJDPS) Vol.4, No.1, January 2013

Wireless sensor networks is useful for several reasons as 1) connectivity as sensor nodes are moving 2) cost fewer node are used to reduced the cost of network 3) reliability as the multi-hop transmission increase the message loss and 4) energy efficient as sensor node send message is collected by sink node and node near to sink more overloaded.

Many studies show that the mobile sink node collect data from the sensors deployed in the field. Previous study exploit that only one mobile sink node travel in sensing area and collect data from the sensors. Mobile sink node collect the data directly using one hope transmission from the sensor or some time using multi-hop transmission. In some cases cluster technique is used for the data collection from sensor. In this technique the mobile sink node collects data from the cluster head and stored data on the Database (Base station).

Data collected from sensor is stored on the gateways then mobile sink gets that data and stored on the Database (Base station) this is again one technique for data collection process and some time data collection is achieved using the routing protocol. Sensor data collection requires all sensing data are collected and forwarded to the base station correctly and accurately, the processing of these data needs the global knowledge and is much more complex. This feature is prevents using data aggregation or fusion techniques to enhance the network performance. As a result, the major traffic in sensor data collection is the reported data from each sensor to the base station.

This review paper shows WSNs with static sink node and WSNs with mobile sink node and manly focused on data collection method which make communication feasible between sink node and sensor nodes. Also This paper is organized as follows the section 2 shows different types of wireless sensor networks architectures used for data collection purpose; section 3 shows the preliminaries in wireless sensor network and data collection with sink nodes, section 4 gives review on data collection process and related work done in data collection process; section 5 find the research gap between various data collection process in WSNs and finally section 6 conclusion depending on above sections.

\section{Wireless Sensor Network ArChitecture}

Wireless Sensor networks are categorized in to 1) layered architecture 2) cluster architecture and 3) sensor nodes with mobile sink node architecture.

To understand Wireless sensor network first understand basic architecture of sensor network that consist of

Sink Node are data collector all sensor nodes send data to the sink node.

Sensor nodes are source information, they may also forward message in network.

Cluster head receives data send by sensor node. 


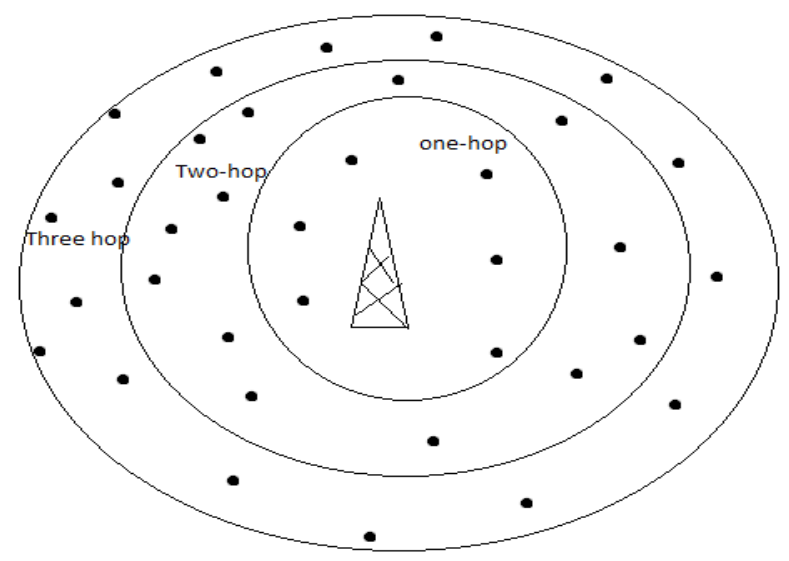

Figure 1. Layered Architecture of wireless sensor network

Above Figure 1 shows layered architecture of WSNs it consist of one sink node and multiple sensors node that sends the data to the sink using one hop, two hop etc.. All information is collected by sink node only.

Cluster architecture consist of sensor nodes clusters each governed by cluster head. The node in each cluster sends the messages to cluster head and these cluster head sends messages to sink node which usually a base station as shown in Figure 2.

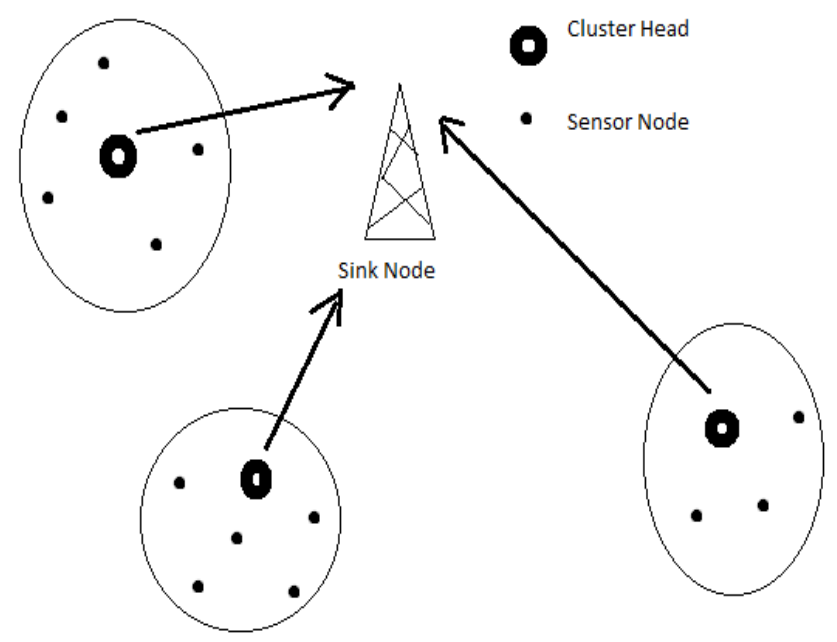

Figure 2. Cluster Architecture of wireless sensor network

Figure 3 shows Wireless Sensor network with mobile sink node architecture in which mobile sink travel in sensing area and collect data from sensors nodes. It consist of Sensor Node, Mobile Sink Node, sometimes it uses Data collector installed within sensing area. 


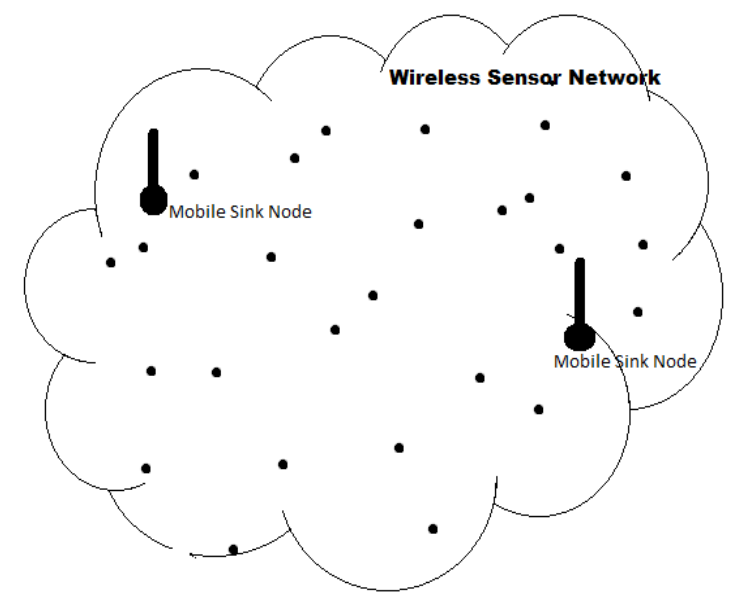

Figure 3. Sensor node with mobile sink node architecture.

Data collection in wireless sensor networks with mobile sink node consist of following approaches

1. Discovery -Mobility independent, knowledge base

2. Data Transfer-Joint discovery and data transfer

3. Routing- Flat, proxy-based.

4. Motion Control-Trajectory -Static, dynamic, Speed and Hybrid.

\section{Preliminaries}

\subsection{Wireless Sensor Network}

A Spatially distributed autonomous sensor to monitor physical or environmental conditions, such as temperature, sound, pressure, etc. is called wireless sensor network (WSN). Applications are Area monitoring Forest fire detection, Air pollution monitoring, Industrial monitoring, Agriculture, Structural monitoring, and smart home monitoring.

\subsection{Data collection in Wireless sensor network}

\subsubsection{Sink node}

All collected sensor data are forwarded to sink node. It is used for data collection purpose it can be a base station or access point.

\subsubsection{Multiple Sink}

In Multiple Sink Node Data Collection Problem data from sensor nodes needs to be transmitted to one of multiple sinks in wireless sensor networks. To minimize the latency of data collection, Schedule designs an approximation algorithm.

\subsubsection{Approximate Data Collection (ADC)}

$\mathrm{ADC}$ is to divide a sensor network into clusters, discover local data correlations on each cluster head, and perform global approximate data collection on the sink node according to model parameters uploaded by cluster heads. 
ternational Journal of Distributed and Parallel Systems (IJDPS) Vol.4, No.1, January 2013

\subsubsection{Using Routing Protocol}

There are various routing protocols are available for data collection in wireless sensor network in [10]. 1) Location based protocol 2) Data Centric Protocols 4) Hierarchical Protocols 5) Mobility-based Protocols 6) Multipath-based Protocols 7) Heterogeneity-based Protocols 8) QoS-based Protocols.

\subsection{Comparison of sink node}

Static sink node was used for data collection in wireless sensor network by using multi hop forwarding so there is more energy consumption nearby node around Base station and relaying in data from other one.

Mobile sink node was used to collect data from sensors node and stored it to the base station. But due to failure of a node whole process fail as only one mobile sink node is using.

Multiple sink nodes were used in data collection process from the sensors and stored collected data on the base station in the database. Here when one of the node mobile sink node fail other is available as the backup one.

\section{RELATED WORK}

\subsection{Sparse Sensor Networks using Data Mules modelling for data collection}

A mobile sink node called data MULE travel in sensing area randomly and collect the data. It collects all the data from all sensors so communication traffic increases. In [1] and [2] Shah, analyzes an architecture in sparse network to collect data from sensors data Mules are present in the environment. Data is collected when Mules is in close range of sensors. Mules collected data drop off to the access point.

\subsection{Clustered Aggregation Technique for data collection}

In cluster aggregation method (CAG) the sensor transmit their data to cluster head that are largely different from the cluster head. CAG is proposed in [3] using spatial correlation of sensors data it reduces the number of transmission and provides result to aggregate queries.

\subsection{Dual-Sink Using Mobile and Static Sinks in WSNs for data collection}

The problem of energy loss during the data collection is removing by proposing the Dual sink node which is distributed and energy efficient protocol for that purpose [4] Wu use both static and mobile sink node.

\subsection{Data Collection in Sensor Networks with Data Mules}

Data collection in sensing network with data Mules is proposed the protocol for reliable and energy efficient data collection [5]. This paper focused on main phases such as data discovery and data transfer.

\subsection{Data Gathering Scheme for WSNs with Static and Mobile Sinks for Energy Efficiency}

To balance the energy consumption among the sensor mobile sink node is used in data collection process. The Problem of energy consumption and large transmission delay is due to the change of sensor route. Static sink node is used with the mobile sink node as in [6]. 
International Journal of Distributed and Parallel Systems (IJDPS) Vol.4, No.1, January 2013

\subsection{Data collection in Sensor Networks with a Mobile Sink for Data Quality Maximization}

The gateways which are used to communicate with mobile sink node deployed nearby trajectories. The data quality can be maximize as mobile sink node moving in sensing area along a fixed trajectory to collect data [7] Liang.

\subsection{Fairness and Data Amount on Sensor Data Collection with a Mobile Sink}

Fair sensor data collection protocol (FSDC) gives upper limit to the first communication time to the sensor. Communication traffic increases when mobile sink goes out of communication rage in [8].

\subsection{A Sensor Data Collection Method with a Mobile Sink for Communication Traffic Reduction}

In [9] Seino, proposed sink node travel in fixed route in sensing area and collect the data from sensors and stored on the base station. Communication traffic can be reduced by delivering the predicated sensor data. In this method the mobile sink node broadcast the predicated value to the sensor and only that sensor can send the data which exceeds the admissible error margin.

\section{RESEARCH GAP}

In [1], Sparse network is used it consist of Mules, Access point, sensors node .For the Data Mules and mobility this model consist of two dimensional random walk. In this model sensor is organized in the form of two dimensional grids and using this technique the short range communication of sensors gives large power saving and Model gives fewer infrastructures, con's are sensor had to wait for mules to collect data therefore latency is the main disadvantage.

In CAG method propose by [3] Yoon, et al. provides the approximate result to aggregation query for the reduction of transmissions. This model provides the lossy CAG algorithm which requires only one value to be participated in aggregation. This Algorithm divided into two parts 1) Query 2) Response and use only one value per cluster. Cluster head is used in aggregation process, main advantage is spatial correlation is used for the improvement of efficiency in wireless sensor network, this save communication overheads up to $70.9 \%$.

Two sink nodes are used for data collection process called Dual Sink proposed by Wu, et al [4]. Dual sink is a protocol for data collection in fully distributed wireless sensor network, uses two node called static sink node and mobile sink node. The algorithm consists of static sink node broadcast Hello message at only one time at the beginning time. Mobile sink node broadcast Hello message but only subset nodes in wireless sensor network and sensor node which processed four messages like hello, data ,EnQry, EnRpl. Which gives a steady lifetime improvement for Wireless sensor network but No lifetime gain is achieved for only one mobile sink node when network size grows to over threshold.

Anastasi et al. [5] proposed the data Mules collect data from sensor deployed in sensing field and stored at access point. Here protocol is developed for the energy efficient and reliable data collection in wireless sensor network. This protocol mainly focused on the discovery and data transfer phase. Data collection process divided into three parts 1) static node perform discovery 2) Mule present data transferred 3) sleep state of static node this improvement in this paper gives a protocol with low duty cycles is very energy efficient, No energy wastage as correct assumption is there for discovery part.

$\mathrm{Xu}$ et al. [6] proposed the wireless sensor network with mobile sink node travel along a fixed trajectory (Route) and gateway is deployed in the sensing field to collect the data from sensor and then it is forwarded to the mobile sink node. This model is divided into three tiers 1) at 
International Journal of Distributed and Parallel Systems (IJDPS) Vol.4, No.1, January 2013

bottom sensor node 2) middle tier gateway 3) top tier mobile sink node. Therefore Energy efficient routing protocol is developed so that mobile sink node efficiently collects data. But deployments of gateway give costly formation of a network.

Data collection method using sink node for communication traffic reduction is proposed Seino et al. [9]. This method consists of the single sink node travel along fixed route and collect data from sensor based on the predicated values. Mobile sink node travel in sensing area and collect sensor data for first round and stored that data on database, but for second round mobile sink node broadcast predicated sensor value to the sensor and only those sensor can send the data which exist admissible error margin called threshold. This Sink node efficiently collects data from sensor and communication traffic reduces but when mobile sink node fail system is not in used for communication and data collection purposed.

Cheng et al. [11] proposed a delay-aware data collection network structure for wireless sensor networks. The main objective of this delay-aware data collection network structure is to minimize delays in the data collection processes of wireless sensor networks. A centralized and a decentralized approach are used two network formation algorithm designed to construct the proposed network structure. This two network formation approaches are derived to provide optimized results for networks with different sizes. The performance of the proposed delayaware data collection network structure is compared with a multiple-cluster two-hop network structure, a single-chain network structure, a minimum spanning tree network structure, and a collection tree network structure. This network structure is most efficient in terms of data collection time among all the network structures. The delay-aware data collection network structure can greatly reduce the data collection time while keeping the total communication distance and the network lifetime at acceptable values.

Again one techniques of data collection in wireless sensor network is Adaptive approximate data collection method. In this technique the adaptive data approximation algorithm should be self-adaptive to the changes of the sensor readings timely as sensor readings change slowly according to the change of physical phenomena. This data approximation algorithm consists of two parts; data approximation learning algorithm and data approximation monitoring algorithm as in [12].

Cheng et al. [13] proposed schemes for data collection with data aggregation for static sink in wireless sensor networks. In this scheme Block partition is used and then each block selects a sensor node as local sink for the data collection of the local area. Local sink then transmit their data to the global sink after aggregating to achieve less energy consumption.

Konstantopoulos et al. [14] proposed Mobicluster protocol for sensor node under the assumption of SNs are location aware. The protocol consist of five phases first clustering approach is used for WSN, second rendezvous nodes (RNs) selection which guarantee connectivity of sensor islands with MS, third cluster head (CHs) Attachment to RNs, fourth Data Aggregation and Forwarding to the RNs and finally Communication between RNs and Mobile Sinks.

\section{CONCLUSIONS}

This paper gives a review on WSNs with sink node and their architecture then shows the comparative study of different sensor data collection method, sink and mobile sink node data collection method it then decides which technique is efficient for the data collection from the sensor nodes. The data collection using routing protocol has some problems related delay, energy consumption. The delay aware data collection network structure reduces data collection time by keeping the total communication distance and the network lifetime at acceptable values. Studying the above literature the data collection based on the Mobile sink node is better than the data collection by using static sink node. 
International Journal of Distributed and Parallel Systems (IJDPS) Vol.4, No.1, January 2013

\section{REFERENCES}

[1] S. Jain, R.C. Shah, S. Roy and W. Brunette, (June 2006) "Exploiting mobility for energy efficient data collection in wireless sensor Networks,"Proc. IEEE Workshop on Modeling and Optimization in Mobile Ad hoc and Wireless Networks (WiOpt'06), vol.11, issue 3, pp.327-339.

[2] R.C. Shah, S. Roy, S. Jain, and W. Brunette (May 2003), "Data MULEs: modeling a three-tier architecture for sparse sensor networks," Proc. Int. Workshop on Sensor Network Protocols and Applications (SNPA'03), pp 30-41.

[3] S.H. Yoon, and C. Shahabi (May 2005), "Exploiting spatial correlation towards an energy efficient clustered aggregation technique (CAG)", Proc. IEEE Int. Conf. Communications (ICC'05), pp.82-98.

[4] Xiaobing Wu, Nanjing Univ., Nanjing Guihai Chen (Aug. 2007), "Dual-Sink: Using Mobile and Static Sinks for Lifetime Improvement in Wireless Sensor Networks ",Computer Communications and Networks, ICCCN 2007, pp 1297 - 1302.

[5] Anastasi, G., Conti, M., Di Francesco, M. (July 2008),'Data collection in sensor networks with data mules: An integrated simulation analysis "Computers and Communications, ISCC 2008. , pp 1096 - 1102 .

[6] Yanbin Weng; Weijia Jia; Guojun Wang (Sept 2010), "Energy efficient data gathering scheme for wireless sensor networks with static and mobile sinks", Network Infrastructure and Digital Content, 2010, pp 748 - 752.

[7] Xu Xu , Sch. of Comput. Sci., Australian Nat. Univ., Canberra, ACT, Australia, Weifa Liang; Wark, T (June 2010), "Data Quality Maximization in Sensor Networks With a Mobile Sink", "Distributed Computing in Sensor Systems and Workshops (DCOSS), 2011 ”,pp 1-8.

[8] Seino, W., Sakashita, S., Yoshihisa, T., Hara, T. and Nishio, S.( Nov. 2010), "A Communication Protocol to Improve Fairness and Data Amount on Sensor Data Collection with a Mobile Sink," Proc. Int. Conf. on Broadband, Wireless Computing, Communication and Applications (BWCCA'2010), pp 33-40.

[9] Seino, W., Sakashita, S., Yoshihisa, T., Hara, T. and Nishio, S.( March 2012), “A Sensor Data Collection Method with a Mobile Sink for Communication Traffic Reduction by Delivering Predicted Values", pp 613-618.

[10] Xiaobo Zhang; Heping Wang; Khokhar, A (2006), “An Energy-Efficient Data Collection Protocol for Mobile Sensor Networks”, Vehicular Technology Conference, VTC-2006, pp 1-5.

[11] Chi-Tsun Cheng, Member, IEEE, Chi K. Tse, Fellow, IEEE, and Francis C. M. Lau, Senior Member, IEEE (March 2011), "A Delay-Aware Data Collection Network Structurefor Wireless Sensor Networks", IEEE Sensors Journal, Vol. 11, No. 3.

[12] Chao Wang, Huadong Ma, Member, IEEE, Yuan He, Member, IEEE, and Shuguagn Xiong (June 2012), “Adaptive Approximate Data Collection for Wireless Sensor Networks", IEEE Transactions on Parallel and Distributed Systems, Vol 23, no.6, pp 1004-1016.

[13] Chia-Hsin Cheng , Yunlin, Taiwan Hsinying Liang ; Yung-Fa Huang ; Tian-Yue Wu (July 2012), "Energy Efficient Block Division Methods for Data Aggregation in Wireless Sensor Networks" , Sixth International conference on Innovative Mobile and Internet Services in Ubiquitous Computing (IMIS), pp $454-458$.

[14] Konstantopoulos, C.; Pantziou, G.; Gavalas, D.; Mpitziopoulos, A.; Mamalis, B. (May 2012), “A Rendezvous-Based Approach Enabling Energy-Efficient Sensory Data Collection with Mobile Sinks", IEEE Transactions on Parallel and Distributed Systems, vol.23, no.5, pp.809-817. 